\title{
REFINED PARAMETERS OF THE PLANET ORBITING HD 189733
}

\author{
G. Á. Bakos, ${ }^{1,2}$ H. Knutson, ${ }^{1}$ F. Pont ${ }^{3}$ C. Moutou,${ }^{4}$ D. Charbonneau, ${ }^{1}$ A. Shporer, ${ }^{5}$ F. Bouchy, ${ }^{6,7}$ \\ M. Everett, ${ }^{8}$ C. Hergenrother, ${ }^{9}$ D. W. Latham, ${ }^{1}$ M. Mayor,${ }^{3}$ T. Mazeh, ${ }^{5}$ R. W. Noyes, ${ }^{1}$ \\ D. Queloz, ${ }^{3}$ A. PÁl, ${ }^{1,10}$ and S. UdRY ${ }^{3}$ \\ Received 2006 March 8; accepted 2006 May 18
}

\begin{abstract}
We report on the $B V R I$ multiband follow-up photometry of the transiting extrasolar planet HD $189733 \mathrm{~b}$. We revise the transit parameters and find a planetary radius of $R_{P}=1.154 \pm 0.033 R_{\mathrm{J}}$ and an inclination of $i_{P}=85^{\circ} .79 \pm 0.24$. The new density $\left(\sim 1 \mathrm{~g} \mathrm{~cm}^{-3}\right)$ is significantly higher than the former estimate $\left(\sim 0.75 \mathrm{~g} \mathrm{~cm}^{-3}\right)$; this shows that from the current sample of nine transiting planets, only HD 209458 (and possibly OGLE-10b) have anomalously large radii and low densities. We note that due to the proximity of its parent star, HD 189733b currently has one of the most precise radius determinations among extrasolar planets. We calculate new ephemerides, $P=2.218573 \pm 0.000020$ days and $T_{0}=2453629.39420 \pm 0.00024$ (HJD), and estimate the timing offsets of the 11 distinct transits with respect to the predictions of a constant orbital period, which can be used to reveal the presence of additional planets in the system.
\end{abstract}

Subject headings: planetary systems — stars: individual (HD 189733)

Online material: color figures, machine-readable table

\section{INTRODUCTION}

HD 189733 is one of nine currently known main-sequence stars orbited by a transiting giant planet. The system is of exceptional interest because it is the closest known transiting planet $(D=19.3 \mathrm{pc})$ and thus is amenable to a host of follow-up observations. The discovery paper by Bouchy et al. (2005, hereafter B05) derived the key physical characteristics of the planet, namely, its mass $\left(1.15 \pm 0.04 M_{\mathrm{J}}\right)$ and radius $\left(1.26 \pm 0.03 R_{\mathrm{J}}\right)$, on the basis of radial velocity observations of the star made with the ELODIE spectrograph at the $1.93 \mathrm{~m}$ telescope at the Observatoire de HauteProvence (OHP), together with photometric measurements of one complete and two partial transits made with the $1.2 \mathrm{~m}$ telescope also at OHP. With these parameters, HD $189733 \mathrm{~b}$ had a large radius, comparable to that of HD 209458b (Laughlin et al. 2005; Wittenmyer et al. 2005; Knutson et al. 2006), and a density roughly equal to that of Saturn $\left(\rho \sim 0.75 \mathrm{~g} \mathrm{~cm}^{-3}\right)$.

Determining precise radii of extrasolar planets in addition to their mass is an important focus of exoplanet research (see, e.g., Bouchy et al. 2004; Torres et al. 2004) because the mean density of the planets can shed light on their internal structure and evolution. According to Baraffe et al. (2005), the radii of all known extrasolar planets are broadly consistent with models, except for HD 209458b. This planet with large radius and low density ( $\rho \sim 0.33 \mathrm{~g} \mathrm{~cm}^{-3}$ ) has attracted considerable interest, and various mechanisms involving heat deposition beneath the surface

\footnotetext{
1 Harvard-Smithsonian Center for Astrophysics, 60 Garden Street, Cambridge, MA 02138; gbakos@cfa.harvard.edu.

${ }_{2}$ Hubble Fellow.

3 Observatoire de Genève, 51 chemin des Maillettes, 1290 Sauverny, Switzerland.

4 Laboratoire d'Astrophysique de Marseille, Traverse du Siphon, 13013 Marseille, France.

5 Wise Observatory, Tel Aviv University, 69978 Tel Aviv, Israel.

6 Observatoire de Haute-Provence, 04870 Saint Michel l'Observatoire, France.

7 Institut d'Astrophysique de Paris, 98bis Boulevard Arago, 75014 Paris, France.

8 Planetary Science Institute, 1700 East Fort Lowell Road, Tucson, AZ 85719.

9 Department of Planetary Sciences and Lunar and Planetary Laboratory, University of Arizona, 1629 East University Boulevard, Tucson, AZ 85721.

${ }^{10}$ Department of Astronomy, Eötvös Loránd University, Pf. 32, 1518 Budapest, Hungary.
}

have been suggested (Laughlin et al. 2005 and references therein). An additional motivation for obtaining accurate planetary radii is proper interpretation of follow-up data, notably secondary eclipse and reflected light observations. This is of particular relevance to HD 189733b, which has been recently observed by the Spitzer Space Telescope (Deming et al. 2006), and where the brightness temperature depends on the radius ratio of the planet to the star.

Both by extending the current, very limited sample of transiting exoplanets, and by precise determination of the physical parameters, it will become possible to refine theoretical models and decide which planets are "typical." Close-by, bright stars, such as the host star of HD 189733, are essential in this undertaking. The OGLE project (Udalski et al. 2002a, 2002b, 2002c) and follow-up observations (e.g., Konacki et al. 2004; Moutou et al. 2004; Pont et al. 2005) made a pivotal contribution to the current sample by the discovery of more than half of the known transiting planets. Follow-up observations, however, are cumbersome due to the faintness of the targets and require the largest available telescopes. The typical errors of mass and radius for these host stars are $\sim 0.06 M_{\odot}$ and $\sim 0.15 R_{\odot}$, and the corresponding errors in planetary parameters are $\sim 0.13 M_{\mathrm{J}}$ and $\sim 0.12 R_{\mathrm{J}}$, respectively. However, for planets orbiting bright stars in the solar neighborhood, errors at the level of a few percent can be reached for both the mass and radius.

In this paper we report a number of follow-up photometric measurements of HD 189733, using six telescopes spaced around the world. Together with the original OHP photometry, we use these measurements to determine revised values for the transit parameters and give new ephemerides. First we describe the follow-up photometry in detail ( $(2)$, followed by the modeling, which leads to the revised estimate of the planetary radius $(\S 3)$, and we conclude the paper in $\S 4$.

\section{OBSERVATIONS AND DATA REDUCTION}

We organized an extensive observing campaign with the goal of acquiring multiband photometric measurements of the transits of HD 189733 caused by the hot Jupiter companion. Including the discovery data of B05 that were obtained at OHP, altogether four sites with seven telescopes contributed data to two full and 
TABLE 1

Summary of Instruments Used in the Observing Campaign of HD 189733

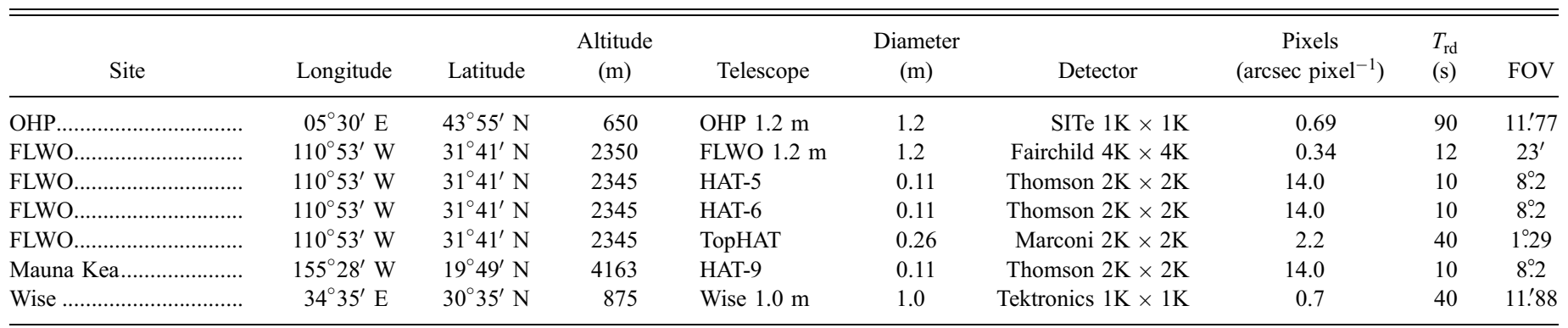

eight partial transits in the Johnson $B, V, R$, and $I$ and the Sloan $r$ photometric bandpasses. The sites and telescopes employed are spread in geographic longitude, which facilitated gathering the large number (close to 3000) of individual data points spanning 2 months.

The following telescopes were involved in the photometric monitoring: the $1 \mathrm{~m}$ telescope at the Wise Observatory, Israel; the $1.2 \mathrm{~m}$ telescope at OHP; the $1.2 \mathrm{~m}$ telescope at the Fred Lawrence Whipple Observatory (FLWO) of the Smithsonian Astrophysical Observatory (SAO); the $0.11 \mathrm{~m}$ HAT-5 and HAT-6 wide-field telescopes plus the $0.26 \mathrm{~m}$ TopHAT telescope, also at FLWO; and the $0.11 \mathrm{~m}$ HAT-9 telescope at the Submillimeter Array site at Mauna Kea, Hawaii. An overview of the sites and telescopes is shown in Table 1.

A summary of the observations is shown in Table 2. The telescopes are identified by the same names as in Table 1 . The transits have been numbered starting with the discovery data, $N_{\text {tr }} \equiv 0$, and are identified later in the text using these numbers. In the following subsections we summarize the observations and reductions that are specific to the sites or instruments.

\subsection{Observations by the OHP $1.2 \mathrm{~m}$ Telescope}

These observations and their reduction were already described in B05. Summarizing briefly, the $1.20 \mathrm{~m} \mathrm{f} / 6$ telescope was used together with a $1 \mathrm{~K}$ back-illuminated CCD having 0 ". 69 pixel $^{-1}$ resolution. Typical exposure times were $6 \mathrm{~s}$ long, followed by a $90 \mathrm{~s}$ readout. The images were slightly defocused, with FWHM $\approx 2$ ". 8 .

Full-transit data were obtained in the Johnson $B$ band under photometric conditions for the $N_{\mathrm{tr}}=0$ transit. This is shown by the "OIBEO" flag in Table 2, indicating that the out-of-transit part before the ingress, the ingress, the bottom, the egress, and the out-of-transit part after the egress have all been observed. This is an important part of the combined data set, as it is the only full transit seen in the $B$ band. In addition, partial-transit data were obtained for the $N_{\text {tr }}=4$ event using the Cousins $R$-band filter $\left(R_{\mathrm{C}}\right)$ under acceptable photometric conditions, as well as

TABLE 2

Summary of HD 189733 Observations

\begin{tabular}{|c|c|c|c|c|c|c|c|c|c|c|}
\hline Telescope & Filter & $N_{\text {tr }}$ & Epoch & $\begin{array}{l}\text { Date } \\
\text { (UT) }\end{array}$ & Transit & Conditions & $\begin{array}{c}\sigma_{\mathrm{OOT}} \\
(\mathrm{mmag})\end{array}$ & $\begin{array}{c}\sigma_{\text {sys }} \\
(\mathrm{mmag})\end{array}$ & $\begin{array}{l}\text { Cadence } \\
\text { (s) }\end{array}$ & $\begin{array}{l}\text { Aperture Radius } \\
\quad(\operatorname{arcsec})\end{array}$ \\
\hline OHP $1.2 \mathrm{~m}$ & $B$ & 0 & 53629.4 & 2005 Sep 15 & OIBEO & 5 & 2.6 & 1.3 & 86 & 10 \\
\hline OHP 1.2 m............................ & $R_{\mathrm{C}}$ & 4 & 53638.3 & 2005 Sep 24 & --BEO & 4 & 3.0 & 1.2 & 95 & 10 \\
\hline OHP $1.2 \mathrm{~m}$ & $R_{\mathrm{C}}$ & 5 & 53640.5 & 2005 Sep 26 & OI--- & 3 & 6.8 & 2.4 & 95 & 10 \\
\hline FLWO $1.2 \mathrm{~m} \ldots \ldots \ldots \ldots . .$. & $r^{\mathrm{a}}$ & 6 & 53642.7 & 2005 Sep 29 & OIBEO & $4^{\mathrm{b}}$ & 2.6 & 0.5 & 17 & 20 \\
\hline TopHAT ............................ & V & 6 & 53642.7 & 2005 Sep 29 & OIBEO & $4^{b}$ & 4.6 & 3.0 & 70 & 10 \\
\hline HAT-9 ........................... & $I_{\mathrm{C}}$ & 7 & 53644.9 & 2005 Oct 1 & OIB-- & 4 & 4.6 & 1.2 & 99 & 42 \\
\hline HAT-9 ........................... & $I_{\mathrm{C}}$ & 16 & 53664.9 & 2005 Oct 21 & OIB-- & 4 & 4.3 & $\ldots$ & 100 & 42 \\
\hline 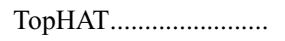 & V & 19 & 53671.6 & 2005 Oct 28 & ---eO & 4 & 5.3 & $\ldots$ & 106 & 10 \\
\hline HAT-5 .......................... & $I_{\mathrm{C}}$ & 19 & 53671.6 & 2005 Oct 28 & ---EO & 4 & 4.6 & $\ldots$ & 103 & 42 \\
\hline HAT-5 ....................... & $I_{\mathrm{C}}$ & 20 & 53673.8 & 2005 Oct 30 & OIb-- & 5 & 3.3 & 0.9 & 85 & 42 \\
\hline Wise $1.0 \mathrm{~m}$ & $B$ & 22 & 53678.2 & 2005 Nov 3 & --bEO & 3 & 5.5 & 1.1 & 49 & 10 \\
\hline
\end{tabular}

Noтеs.-The table summarizes all observations that were part of the observing campaign described in this paper. Not all of them were used for refining the ephemerides or parameters of the transit; see Tables 3 and 5 for reference. The $N_{\text {tr }}$ column shows the number of transits since the discovery data. The Epoch and Date columns show the approximate time of midtransit. The Transit column describes in a terse format which parts of the transits were observed: Out-of-Transit (OOT) section before the transit, Ingress, Bottom, Egress, and OOT after the transit (small letters indicate marginal observation of the given transit phase). Missing sections are shown by hyphens. The Conditions column indicates the photometric conditions on a scale of $1-5$, where 5 is absolute photometric, 4 is photometric most of the time with occasional cirrus/fog (relative photometric), 3 indicates broken cirrus, and 2 indicates poor conditions. The $\sigma_{\mathrm{OOT}}$ column gives the rms of the OOT section at the cadence shown in the next column. If the transit was full, the value of $\sigma_{\text {OOT }}$ was computed separately from the pre- and posttransit data, and the smaller value is shown. The $\sigma_{\text {sys }}$ column shows the estimated amplitude of systematics (for details, see $\S 3.2$ ). The Aperture Radius column shows the aperture radius used in the photometry, in units of arcseconds.

${ }^{a}$ Sloan $r$ filter.

${ }^{\mathrm{b}}$ Conditions were nonphotometric before the transit (on the initial part of the OOT), became photometric for the entire duration of the transit, and deteriorated after the transit at the end of the OOT. 

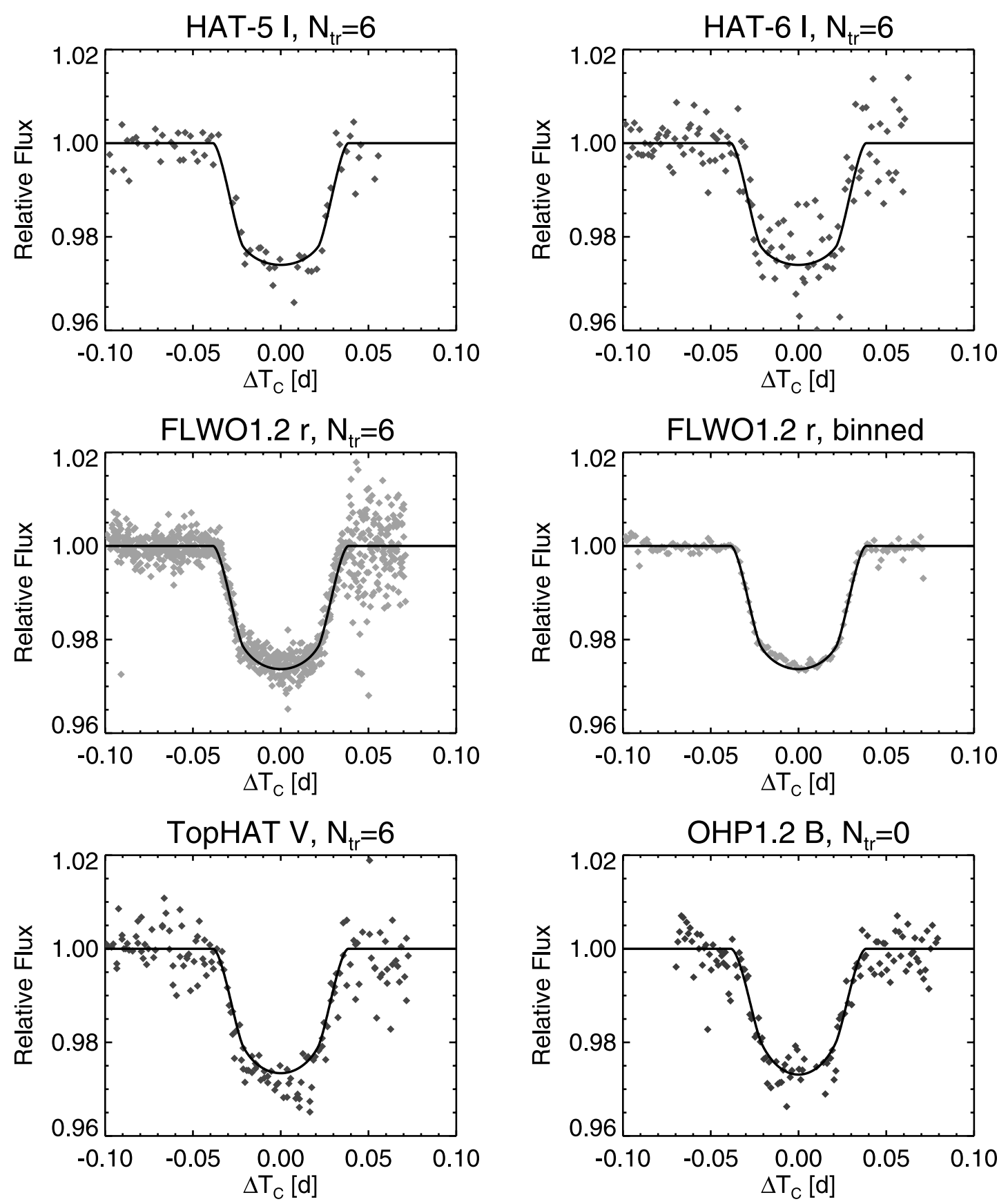

FIG. 1.- Five full eclipses examined in this work, with best-fit transit curves overplotted. The figure in the electronic edition is color-coded according to the bandpass used. [See the electronic edition of the Journal for a color version of this figure.]

for the $N_{\text {tr }}=5$ event, using the same filter under nonphotometric conditions. The frames were subject to the bias, dark, and flatfield calibration procedure, followed by cosmic-ray removal. Aperture photometry was performed in an aperture of 9." 6 radius, using the DAOPHOT (Stetson 1987) package.

The $B$-band light curve published in the B05 discovery paper used the single comparison star HD 345459. This light curve suffered from a strong residual trend, as suggested by the $\sim 0.01 \mathrm{mag}$ difference between the pre- and posttransit sections. This trend was probably a consequence of differential atmospheric extinction and was removed by a linear air-mass correction, bringing the two sections to the same mean value. This ad hoc correction, however, may have introduced an error in the transit depth. In this paper, we used six comparison stars in the field of view (se- lected to have comparable relative flux to HD 189733 before and after transit). A reference light curve was built by co-adding the normalized flux of all six stars and was subtracted from the normalized light curve of HD 189733. The new reduction shows a residual out-of-transit (OOT) slope 4.2 times smaller than in the earlier reduction. The resulting transit depth in the $B$ band is decreased by about $20 \%$ compared to the discovery data. This illustrates the large contribution of photometric systematics that must be accounted for in this kind of measurement. The $R$-band data set is not as sensitive to the extinction effect as the $B$ band; hence, the selection of comparison stars has a minimal impact on the shape of the transit curve. The $B$-band light curve is shown in the bottom right panel of Figure 1 , and the $R$-band light curves are exhibited in the top panels of Figure 2. 

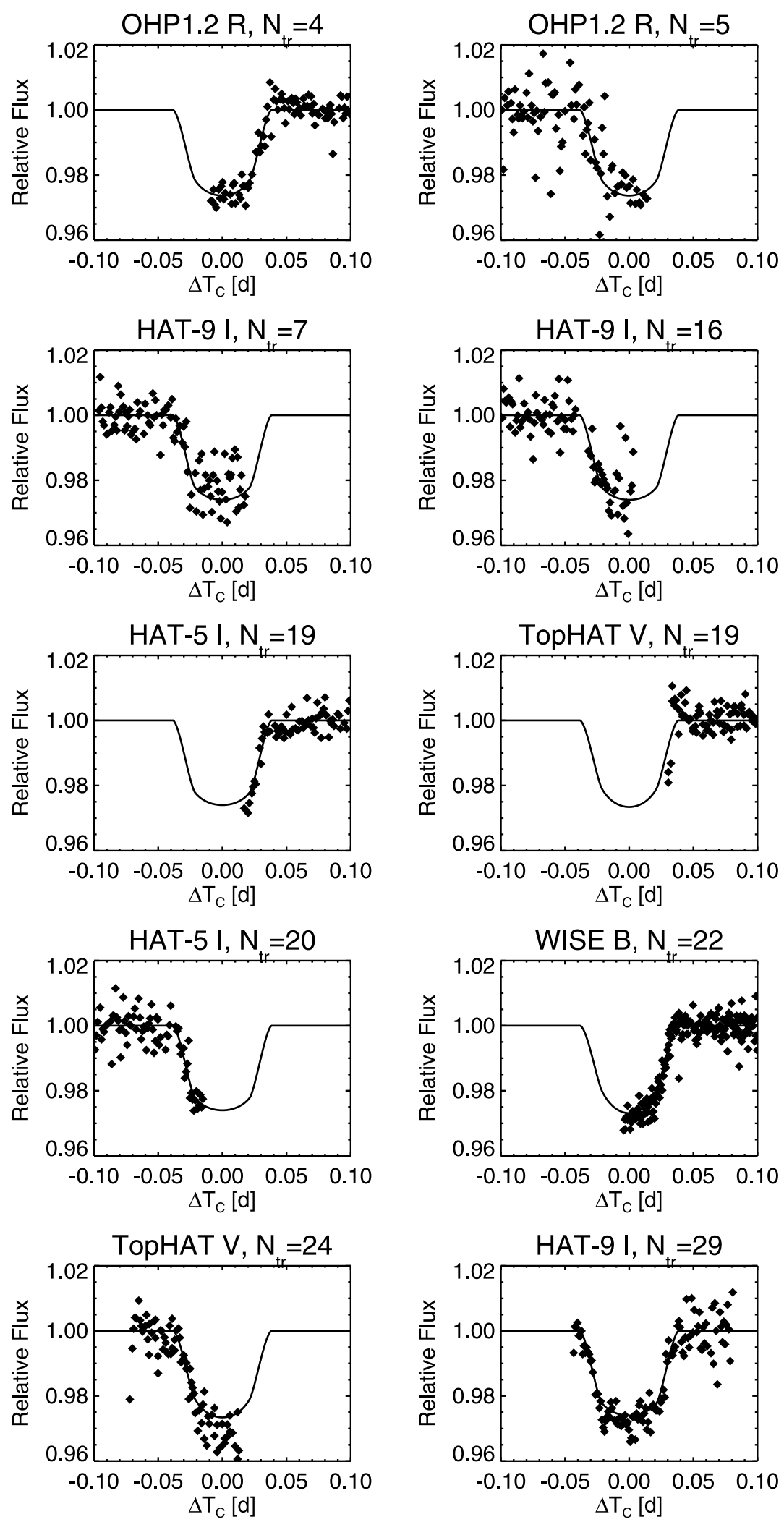

FIG. 2.-Ten partial eclipses examined in this work, with best-fit transit curves overplotted. The eclipses are listed sequentially by date, from top left to bottom right. These eclipses were not used in the fit for the planetary radius, inclination, stellar mass, and stellar radius. The figure in the electronic edition is color-coded according to the bandpass used. [See the electronic edition of the Journal for a color version of this figure.] 


\subsection{Observations by the FLWO $1.2 \mathrm{~m}$ Telescope}

We used the FLWO $1.2 \mathrm{~m}$ telescope to observe the full transit of $N_{\mathrm{tr}}=6$ in the Sloan $r$ band. The detector was Keplercam, which is a single-chip $4 \mathrm{~K} \mathrm{CCD}$ with $15 \mu \mathrm{m}$ pixels that correspond to 0 "'34 on the sky. The entire field of view (FOV) is 23'. The chip is read out by four amplifiers, yielding a $12 \mathrm{~s}$ readout with the $2 \times 2$ binning we used. The single-chip design, wide field of view, high sensitivity, and fast readout make this instrument well suited for high-quality photometry follow-up.

The target was deliberately defocused in order to allow longer exposure times without saturating the pixels and to smear out the interpixel variations that may remain after flat-field calibration. The intrinsic FWHM was $\sim 2^{\prime \prime}$, which was defocused to $\sim 10^{\prime \prime}$. While conditions during the transit were photometric, ${ }^{11}$ there were partial clouds before and after. The focus setting was changed twice during the night: first when the clouds cleared, and second, when the seeing improved. In both cases the reason was to keep the signal level within the linear response range of the CCD. We used a large enough aperture that these focus changes did not affect the photometry. All exposures were $5 \mathrm{~s}$ in length, with $12 \mathrm{~s}$ of readout and overhead time between exposures. We observed the target in a single band so as to maximize the cadence and to eliminate flat-fielding errors that might originate from the imperfect subpixel repositioning of the filter wheel. Auto-guiding was used to further minimize systematic errors that originate from the star drifting away on the CCD chip and falling on pixels with different (and not perfectly calibrated) characteristics.

\subsubsection{Reduction and Photometry}

All images were reduced in the same manner: applying overscan correction, subtraction of the two-dimensional residual bias pattern, and correction for shutter effects. Finally, we flattened each image using a combined and normalized set of twilight sky flat images. There was a drift of only $\sim 3^{\prime \prime}$ in pointing during the night, so any large-scale flat-fielding errors were negligible.

To produce a transit light curve, we chose one image as an astrometric reference and identified star centers for HD 189733 and 23 other bright and relatively uncrowded stars in the field. We measured the flux of each star around a fixed pixel center derived from an astrometric fit to the reference stars in a $20^{\prime \prime}$ radius circular aperture using DAOPHOT/PHOT within IRAF $^{12}$ (Tody 1986, 1993) and estimated the sky using the sigma-rejected mode in an annulus defined around each star with inner and outer radii of $33^{\prime \prime}$ and $60^{\prime \prime}$, respectively.

We calculated the extinction correction on the basis of a weighted mean flux of comparison stars and applied this correction to each of our stars. We iteratively selected our comparison stars by removing any that showed unusually noisy or variable trends in their differential light curves. In addition, a few exposures at the beginning and very end of our observing sequence were removed because those observations were made through particularly thick clouds. The resulting light curve represents the observed counts for the star corrected for extinction using a group of six comparison stars within $6^{\prime}$ separation from HD 189733. The light curve is shown in the middle panels of Figure 1.

\footnotetext{
11 This was confirmed from the all-sky Web camera movies taken at the Multiple Mirror Telescope (MMT), which are archived on a nightly basis ( http:// skycam.mmto.arizona.edu).

${ }^{12}$ IRAF is distributed by the National Optical Astronomy Observatory, which is operated by the Association of Universities for Research in Astronomy, Inc., under cooperative agreement with the National Science Foundation.
}

\subsection{Observations by the HAT Network}

An instrument description of the wide-field Hungarian Automated Telescope (HAT) telescopes was given in Bakos et al. (2002, 2004). Here we briefly recall the relevant system parameters. A HAT instrument contains a fast focal ratio (f/1.8) $0.11 \mathrm{~m}$ diameter Canon lens and Peltier-cooled CCD with a frontilluminated $2 \mathrm{~K}$ chip having a $14 \mu \mathrm{m}$ pixel size. The resulting FOV is $8^{\circ} .2$ with a $14^{\prime \prime}$ pixel scale. Using a PSF-broadening technique (Bakos et al. 2004), a careful calibration procedure, and robust differential photometry, the HAT telescopes can achieve 3 mmag precision (rms) light curves at $300 \mathrm{~s}$ resolution for bright stars (at $I \approx 8$ ). The HAT instruments are operated in autonomous mode and carry out robotic observations every clear night.

We have set up a longitude-separated, two-site network of six HAT instruments, with the primary goal being detection of planetary transits in front of bright stars. The two sites are at FLWO, in Arizona, the same site at which the $1.2 \mathrm{~m}$ telescope is located $(\S 2.2)$, and on the roof of the Submillimeter Array atop Mauna Kea, Hawaii (MK).

In addition to the wide-field HAT instruments, we developed a dedicated photometry follow-up instrument called TopHAT, which is installed at FLWO. A brief system description was given in Charbonneau et al. (2006) in context of the photometry follow-up of the HD 149026 planetary transit. This telescope has $0.26 \mathrm{~m}$ diameter, with f/5 Ritchey-Crétien design and a Baker wide-field corrector. The CCD is a $2 \mathrm{~K}$ Marconi chip with $13.5 \mu \mathrm{m}$ pixel size. The resulting FOV is $1^{\circ} .3$ with 2 ". 2 pixel resolution. Similarly to the HATs, TopHAT is fully automated.

Selected stations of the HAT network, along with TopHAT, observed one full and six partial transits of HD 189733 (for details, see Table 2). Observing conditions of the full-transit event at FLWO at $N_{\text {tr }}=6$ have been summarized in $\S 2.2$. This transit was observed by HAT- 5 and HAT- 6 (both in the $I$ band) and by TopHAT (in the $V$ band). The partial-transit observations at numerous later epochs included HAT-5 (FLWO, $I$ band), HAT-9 (MK, $I$ band), and TopHAT (FLWO, $V$ band). Typical exposure times for the wide-field instruments were $60-90 \mathrm{~s}$ with $10 \mathrm{~s}$ readout. TopHAT exposures were $\sim 12 \mathrm{~s}$ long with up to $40 \mathrm{~s}$ readout and download time. All observations were made at slight defocusing and using the PSF-broadening technique. The stellar profiles were 2.5 pixels $\left(35^{\prime \prime}\right)$ and 4.5 pixels $(9.9)$ wide for the HATs and TopHAT, respectively. Although we have no auto-guiding, real-time astrometry was performed after the exposures, and the telescope's position was kept constant with $20^{\prime \prime}$ accuracy.

\subsubsection{Reduction and Photometry}

All HAT and TopHAT images were subject to overscan correction, two-dimensional residual bias pattern and dark subtraction, and normalization with a master sky flat frame. Bias, dark, and sky flat calibration frames were taken each night by each telescope, and all object frames were corrected with the master calibration images that belonged to the specific observing session. Saturated pixels were masked before the calibration procedure.

We used the 2MASS All-Sky Catalog of Point Sources (Skrutskie et al. 2006) as an input astrometric catalog, where the quoted precision is 120 mas for bright sources. A fourth-order polynomial fit was used to transform the Two Micron All Sky Survey (2MASS) positions to the reference frame of the individual images. The typical rms of the transformations was 700 mas for the wide-field instruments and 150 mas for TopHAT.

Fixed center aperture photometry was performed for all these stars. For the wide-field HAT telescopes we used an $r_{\text {ap }}=3$ pixel $\left(42^{\prime \prime}\right)$ aperture, surrounded by an annulus with inner and outer 
TABLE 3

The Light Curve of HD 189733

\begin{tabular}{|c|c|c|c|c|c|c|c|c|c|c|c|}
\hline Tel. & Filt. & $N_{\text {tr }}$ & HJD & Mag & $\begin{array}{l}\text { Merr } \\
\text { (mag) }\end{array}$ & FR & $\mathrm{FR}_{\text {corr }}$ & $\mathrm{FR}_{\text {err }}$ & $\begin{array}{l}\Delta M_{\mathrm{ext}} \\
(\mathrm{mag})\end{array}$ & $\begin{array}{c}\sigma_{\mathrm{mfit}} \\
(\mathrm{mag})\end{array}$ & Qflag \\
\hline OHP1.2......... & $B$ & 0 & 2453629.3205430 & 8.6062 & $\ldots$ & 0.99614 & 0.99612 & $\ldots$ & $\ldots$ & $\ldots$ & $\ldots$ \\
\hline HAT-5 ........... & $I$ & 6 & 2453642.5903353 & 6.7452 & 0.0017 & 0.99522 & 0.99511 & 0.00156 & -0.147 & 0.0142 & G \\
\hline HAT- $6 \ldots \ldots \ldots . . .$. & $I$ & 6 & 2453642.6082715 & 6.7357 & 0.0017 & 1.00397 & 1.00406 & 0.00156 & -0.011 & 0.0160 & G \\
\hline ТорНАТ........ & $V$ & 6 & 2453642.6042285 & 7.6717 & 0.0009 & 0.99844 & 0.99841 & 0.00083 & -0.097 & 0.0080 & G \\
\hline
\end{tabular}

Notes.-Table 3 is presented in its entirety (2938 lines) in the electronic edition of the Astrophysical Journal. A portion is shown here for guidance regarding its form and content, with a sample line for each telescope in the order that they observed a transit with an OOT section. The $N_{\text {tr }}$ column gives the number of transits since the discovery data by OHP on HJD $=2453629.3$. Values in the Mag (magnitude) column have been derived by shifting the zero point of the particular data set at $N_{\mathrm{tr}}$ to bring the median of the OOT section to the standard magnitude value in the literature. Merr ( $\left.\mathrm{FR}_{\mathrm{err}}\right)$ denote the formal magnitude (flux ratio) error estimates based on the photon and background noise (not available for all data). The flux ratio, FR, shows the ratio of the individual flux measurements to the sigma-clipped median value of the OOT at that particular $N_{\text {tr }}$ transit observation. The merger-corrected flux ratio, $\mathrm{FR}_{\text {corr }}$, is described in detail in $\S 2.6$. The $\Delta M_{\text {ext }}$ column gives a measure of the extinction on a relative scale (instrumental magnitude of reference minus image), and the $\sigma_{\text {mfit }}$ column gives the rms of the magnitude fit between the reference and the given frame. Both of these quantities are useful measures of the photometric conditions. The Qflag column gives the quality flag: "G" means good, and "C" indicates that the measurement should be used with caution; for example, the star was marked as saturated. Fits of the transit parameters were performed using the HJD, FR, FR ${ }_{\text {corr }}$, and $\mathrm{FR}_{\mathrm{err}}$ columns.

radii of $r_{1}=5$ pixels $\left(70^{\prime \prime}\right)$ and $r_{2}=13$ pixels $\left(3^{\prime}\right)$, respectively. For TopHAT, the best aperture was $r_{\mathrm{ap}}=5$ pixels $\left(10^{\prime \prime} 8\right)$ with $r_{1}=13$ pixels $\left(29^{\prime \prime}\right)$ and $r_{2}=21$ pixels $\left(46^{\prime \prime}\right)$. The apertures were small enough to exclude any bright neighboring star.

A high-quality reference frame was selected for the wide-field HAT telescopes from the Mauna Kea HAT-9 data, and a separate frame was selected for TopHAT. Because the HAT wide-field instruments are almost identical, we were able to use the HAT-9 reference frame to transform the instrumental magnitudes of HAT-5 and HAT- 6 data to a common system. For this, we used fourthorder polynomials of the magnitude differences as a function of $X$ and $Y$ pixel positions. In effect, we thereby used $\sim 3000$ and $\sim 800$ selected nonvariable comparison stars for the HATs and for TopHAT, respectively. This contributes to the achieved precision, which is only slightly inferior to the precision achieved by the bigger diameter telescopes.

The amount of magnitude correction for HD 189733 between the reference and the individual images is shown in the $\Delta M_{\text {ext }}$ column of Table 3. The same table also indicates the rms of these magnitude fits in the $\sigma_{\text {mfit }}$ column. Both quantities are useful for further cleaning of the data. Because HD 189733 is a bright source, it was saturated on a small fraction of the frames. Saturated data points were flagged in the light curves and were deselected from the subsequent analysis (flagged as " $\mathrm{C}$ " in Table 3).

After cleaning outliers by automatically deselecting points for which the rms of the magnitude transformations was above a critical threshold (typically $25 \mathrm{mmag}$ ), the light curves reached a precision of $\sim 4$ mmag at $90 \mathrm{~s}$ resolution for both the HATs and TopHAT. Full-transit data are shown in the top left, top right, and bottom left panels of Figure 1, and partial-transit data are shown in Figure 2.

\subsection{Observations by the Wise $1.0 \mathrm{~m}$ Telescope}

The Wise $1 \mathrm{~m} \mathrm{f} / 7$ telescope was used to observe the $N_{\mathrm{tr}}=4$ and $N_{\text {tr }}=22$ transits in the $B$ band. The CCD was a $1 \mathrm{~K}$ Tektronics chip with $24 \mu \mathrm{m}$ pixel size that corresponds to 0.696 pixel $^{-1}$ resolution on the sky and a FOV of 11' 88 . The photometric conditions were acceptable on both nights, with FWHM $\approx 2^{\prime \prime}$. Auto-guiding was used during the observations. Frames were calibrated in a similar manner to the FLWO $1.2 \mathrm{~m}$ observations, using twilight flats, and aperture photometry was performed with DAOPHOT.
Unfortunately, OOT data of the first transit $\left(N_{\mathrm{tr}}=4\right)$ (which was also observed from OHP in the $R$ band) are missing, so it is impossible to obtain useful normalization or to apply extinction correction to the transit curve. The second transit $\left(N_{\text {tr }}=22\right)$ was processed using an aperture of $10^{\prime \prime}$ encircled by an annulus with inner and outer radii of $15^{\prime \prime}$ and $25^{\prime \prime}$, respectively. Seven comparison stars were used, all of them bright, isolated, and far from the boundary of the FOV. Extinction correction, derived from the OOT points only, was applied to the resulting stellar light curve. The final curve of this transit is plotted in Figure 2.

\subsection{The Resulting Light Curve}

All photometry originating from the individual telescopes that contains significant OOT data has been merged and is presented in Table 3. We give both the ratio of the observed flux to the OOT flux of HD 189733 ("FR") and magnitudes that are very close to the standard Johnson/Cousins system ("Mag"). Due to the different observing conditions, instruments, photometry parameters (primarily the aperture), and various systematic effects (changing FWHM), the zero points of the observations were slightly offset. Even for the same instrument, filter setup, and magnitude reference frame, the zero points in the flat OOT section were seen to differ by $0.03 \mathrm{mag}$. The offset can be explained by long-term systematic variations and by intrinsic variation of HD 189733 .

In order to correct for the offsets, for each transit observation (as indicated by $N_{\mathrm{tr}}$ in Table 3) we calculated both the median value from the OOT section by rejecting outliers and also the rms around the median. The OOT median was used for two purposes. First, we normalized the flux values of the given light-curve segment at $N_{\text {tr }}$, which are shown in the FR (flux ratio) column of Table 3. Second, we shifted the magnitudes to the standard system in order to present reasonable values in the Mag column. For the standard system we used the Hipparcos values, except for the $R$ band, which was derived by assuming $R-I=0.48$ from Cox (2000).

The formal magnitude errors that are given in the "Merr" column are based on the photon noise of the source and the background noise (e.g., Newberry 1991). They are in a self-consistent system, but they underestimate the real errors, which have contributions from other noise factors, such as (1) scintillation (Young 1967; Gilliland \& Brown 1988), (2) calibration frames (Newberry 
1991), and (3) magnitude transformations depending on the reference stars and imperfectly corrected extinction (indicators of this error source are the $\Delta M_{\text {ext }}$ extinction and the $\sigma_{\text {mfit }}$ rms of extinction corrections in Table 3 ). Because it is rather difficult to calculate these factors, we assumed that the observed rms in the OOT section of the light curves is a relevant measure of the overall noise, and we used this to normalize the error estimates of the individual flux ratios (the "FRerr" column; see below in $\S 2.6$ ).

\subsection{Merger Analysis}

HD 189733 has a number of faint, close-by neighbors that can distort the light curve and may bias the derived physical parameters. These blends can have the following second-order effects: (1) the measured transit will appear shallower, as if the planetary radius was smaller; (2) the depth and shape of the transit will be color-dependent in a different way than one would expect from limb-darkening models; (3) differential extinction can yield an asymmetric light curve; (4) variability of a faint blend can influence the observed light curve. Our goal was to calculate the additional flux in the various apertures and bandpasses shown in Table 2 and to correct our observed flux ratios ( Table 3, in the FR column) to a realistic flux ratio $\left(\mathrm{FR}_{\text {corr }}\right)$.

The 2MASS point-source catalog (Skrutskie et al. 2006) lists some 30 stars within $45^{\prime \prime}$, which is the aperture used at the HAT-5, HAT-6, and HAT-9 telescopes, 5 stars within 20" (FLWO $1.2 \mathrm{~m}$ ), and 3 stars within $15^{\prime \prime}$, which may affect the measurements of the $10^{\prime \prime}$ apertures of OHP $1.2 \mathrm{~m}$, Wise $1.0 \mathrm{~m}$, and TopHAT (apertures are listed in Table 2). To check the reality of listed blends and to search for additional merger stars, we inspected the following sources: the Palomar Observatory Sky Survey (POSS I) red plates (epoch 1951), the Palomar Quick-V Survey (QuickV, epoch 1982), the Second Palomar Sky Survey (POSS II) plates (epoch 19901996), the $2 \mathrm{MASS} J, H$, and $K_{s}$ scans (epoch 2000), and our own images.

We can use the fact that HD 189733 is a high proper motion star with velocity of $\sim 0$ "' $25 \mathrm{yr}^{-1}$ pointing south. It was $\sim 13^{\prime \prime}$ to the north on the POSS I plates and $\sim 4^{\prime \prime}$ to the north on the POSS II plates; thus, we can check its present place when it was not hidden by the glare of HD 189733. The analysis is complicated by the saturation, diffraction spikes, and the limited scan resolution $\left(1{ }^{\prime \prime} 7\right.$ pixel $\left.^{-1}\right)$ of POSS I, but we can confirm that there is no significant source at the epoch 2005 position of HD 189733 down to $\sim 4$ mag fainter in the $R$ band. The reality of all the 2MASS entries was double-checked on the POSS frames.

There are only two additional faint sources that are missing from the 2MASS point-source catalog but were detected by our star extraction on the 2MASS $J, H$, and $K$ scans, the first at $\alpha=20^{\mathrm{h}} 00^{\mathrm{m}} 45^{\mathrm{s}} .12, \delta=+22^{\circ} 42^{\prime} 36^{\prime \prime} .5$ and the second at $\alpha=$ $20^{\mathrm{h}} 00^{\mathrm{m}} 43^{\mathrm{s}} .20, \delta=+22^{\circ} 42^{\prime} 42^{\prime \prime} .5(\mathrm{~J} 2000.0)$. We made sure that these sources were not filter glints or persistence effects on the 2MASS scans; they are also visible on the POSS frames. Their instrumental magnitude was transformed to the $J H K_{s}$ system using the other stars in the field that are identified in the pointsource catalog.

A rough linear transformation was derived between the 2MASS $J, H$, and $K_{s}$ colors and the Johnson/Cousins $B, V, R$, and $I$ by cross-identifying $\sim 450$ Landolt (1992) standard stars and performing linear regression. The uncertainty in the transformation can be as large as $0.1 \mathrm{mag}$, but this is adequate for the purpose of estimating the extra flux (in $B V R I$ ), which is only about a few percent that of HD 189733.

We find that the extra flux in a $45^{\prime \prime}$ aperture is $\delta=1.012,1.016$, 1.018 , and 1.022 times the flux of HD 189733 in the $B, V, R$, and $I$ bands, respectively. The dominant contribution comes from the red star 2MASS $20004297+2242342$ at 11 ".5 distance, which is 4.5 mag fainter. This star has been found (Bakos et al. 2006) to be a physical companion to HD 189733 and thus may also be called HD 189733B (not to be confused with HD 189733b). For the $10^{\prime \prime}$ aperture we assumed that half the flux of HD 189733B is within the aperture. The same $\delta$ flux contribution in the $10^{\prime \prime}$ aperture is $1.003,1.005,1.006$, and 1.008 in $B, V, R$, and $I$, respectively. The corrected flux ratios of the individual measurements to the median of the OOT were calculated in the manner $\mathrm{FR}_{\text {corr }}=$ $1+\delta(\mathrm{FR}-1)$ and are shown in Table 3 . There is a small difference $(\sim 2 \%)$ between the $10^{\prime \prime}$ and $45^{\prime \prime}$ flux contribution; thus, we expect that the former measurements (OHP $1.2 \mathrm{~m}$, Wise $1.0 \mathrm{~m}$, TopHAT) show slightly deeper transits (corrected for blending) than the FLWO $1.2 \mathrm{~m}(r)$ and wide-field HAT network telescopes $(I)$.

\section{DERIVING THE PHYSICAL PARAMETERS OF THE SYSTEM}

We use the full analytic formula for nonlinear limb darkening given in Mandel \& Agol (2002) to calculate our transit curves. In addition to the orbital period and limb-darkening coefficients, these curves are a function of four variables, including the mass $\left(M_{*}\right)$ and radius $\left(R_{*}\right)$ of the star, the radius of the planet $\left(R_{P}\right)$, and the inclination of the planet's orbit relative to the observer $\left(i_{P}\right)$. Because these parameters are degenerate in the transit curve, we use $M_{*}=0.82 \pm 0.03 M_{\odot}$ from B05 to break the degeneracy. As regards $R_{*}$, there are two possible approaches: (1) assume a fixed value from independent measurements $(\S 3.1)$, or $(2)$ measure the radius of the star directly from the transit curve; that is, leave it to vary freely in the fit. Our final results are based on detailed analysis ( $§ 3.2)$ using the first approach. To fully trust the second approach, one would need high-precision data with relatively small systematic errors. Nevertheless, in order to check consistency, we also performed an analysis where the stellar radius was left as a variable in the fit and also checked the effect of systematic variations in the light curves (see below in $\S 3.2$ ). Refined ephemerides and center of transit time residuals are discussed in $\S 3.3$.

\subsection{The Radius of HD 189733}

Because the value of the stellar radius we use in our fit linearly affects the size of the planetary radius we obtain, we use several independent methods to check its value and uncertainty.

First method.-For our first calculation we use 2MASS (Skrutskie et al. 2006) and Hipparcos photometry (Perryman et al. 1997 ) to find the $V$-band magnitude and $V-K$ colors of the star, and we use the relation described in Kervella et al. (2004) to find the angular size of the star. Because this relation was derived using Johnson magnitudes, we first convert the 2MASS $K_{s}=5.541 \pm$ 0.021 magnitude to the Bessell-Brett homogenized system, which in turn is based on the SAAO system and thus is the closest to Johnson magnitude available (Carpenter 2001). We obtain a value of $K=5.59 \pm 0.05$. Most of the error comes from the uncertainty in the $J-K_{s}$ color, which is used in the conversion.

The Johnson $V$-band magnitude from Hipparcos is $7.67 \pm$ 0.01 . This gives a $V-K$ color of $2.09 \pm 0.06$. From Kervella et al. (2004), the limb-darkened angular size of a dwarf star is related to its $K$ magnitude and $V-K$ color by

$$
\log \theta=0.0755(V-K)+0.5170-0.2 K \text {. }
$$

Given the proximity of HD 189733 (19.3 $\pm 0.3 \mathrm{pc})$, reddening can be neglected, despite its low Galactic latitude. The relation 
gives an angular size of $0.36 \pm 0.02$ mas for the stellar photosphere, where the error estimate originates from the errors of $V-K$ and $K$. The small dispersion of the relation was not taken into account in the error estimate, as it was determined by Kervella et al. (2004), who used a fit to a sample of stars with known angular diameters, to be less than $1 \%$. Using the Hipparcos parallax, we find that $R_{*}=0.75 \pm 0.05 R_{\odot}$.

Second method.- We also derive the radius of the star directly from the Hipparcos parallax, the $V$-band magnitude, and the temperature of the star. We first convert from apparent magnitude to absolute magnitude and apply a bolometric correction (Bessell et al. 1998). To solve for the radius of the star, we use the relation

$$
M_{b}=4.74-2.5 \log \left[\left(\frac{T_{\mathrm{eff}, *}}{T_{\mathrm{eff}, \odot}}\right)^{4}\left(\frac{R_{*}}{R_{\odot}}\right)^{2}\right] .
$$

For an effective temperature of $T_{\text {eff, } *}=5050 \pm 50 \mathrm{~K}$ (B05), we measure a radius of $0.74 \pm 0.03 R_{\odot}$.

Third method: isochrones.-An additional test on the stellar radius and its uncertainty comes from stellar evolution models. We find from the Girardi et al. (2002) models that the isochrone gridpoints in the $\left(T_{\text {eff }}, \log g\right)$ plane that are closest to the observed values $\left(T_{\mathrm{eff}, *}=5050 \pm 50 \mathrm{~K}, \log g=4.53 \pm 0.14\right)$ prefer slightly evolved models with $M_{*} \approx 0.80 M_{\odot}$ and $R_{*} \approx 0.79 R_{\odot}$. Alternatively, the Hipparcos $V=7.67 \pm 0.01$ magnitude combined with the $m-M=-1.423 \pm 0.035$ distance modulus yields an absolute $V$ magnitude of $M_{V}=6.25 \pm 0.04$, and the closest isochrone gridpoints prefer less evolved stars with $M_{*} \approx 0.80 M_{\odot}$ and $R_{*} \approx 0.76 R_{\odot}$. The discrepancy between the above two approaches decreases if we adopt a slightly larger distance modulus. Finally, comparison to the Baraffe et al. (1998) isochrones yields $M_{*} \approx 0.80 M_{\odot}$ and $R_{*} \approx 0.76 R_{\odot}$. From isochrone fitting, the error on the stellar radius can be as large as $0.03 R_{\odot}$.

Fourth method.-Recently Masana et al. (2006) calibrated the effective temperatures, angular semidiameters, and bolometric corrections for F-, G-, and K-type stars on the basis of $V$ and 2MASS infrared photometry. They provide, among other parameters, angular semidiameters and radii for a large sample of Hipparcos stars. For HD 189733 they derived $R=0.758 \pm 0.016 R_{\odot}$.

Summary.-Altogether, the various methods point to a stellar radius in the range of $0.74-0.79 R_{\odot}$, with a mean value of $\sim 0.76 R_{\odot}$. In the subsequent analysis we accept the Masana et al. (2006) value of $0.758 \pm 0.016 R_{\odot}$.

\subsection{Fitting the Transit Curve}

We set the mass and radius of the star equal to $0.82 \pm 0.03 M_{\odot}$ and $0.758 \pm 0.016 R_{\odot}$, respectively, and fit for the planet's radius and orbital inclination. The goodness-of-fit parameter is given by

$$
\chi^{2}=\sum_{i=1}^{N}\left(\frac{p_{i}-m_{i}}{\sigma_{m, i}}\right)^{2}
$$

where $m_{i}$ is the $i$ th measured value for the flux from the star (with the median of the OOT points normalized to 1$), p_{i}$ is the predicted value for the flux from the theoretical transit curve, and $\sigma_{m, i}$ is the error for each flux measurement.

For the OHP $1.2 \mathrm{~m}$ and Wise data, where independent errors for each flux measurement are not available, we set the $\sigma_{m, i}$ errors on all points equal to the standard deviation of the OOT points. For the FLWO 1.2 m, HAT, and TopHAT data, where relative errors for individual points are available, we set the median error equal to the standard deviation of the OOT points and use that to
TABLE 4

\begin{tabular}{|c|c|}
\hline Parameter & Best-Fit Value \\
\hline$R_{P}\left(\mathrm{R}_{\mathrm{J}}\right) \ldots \ldots \ldots \ldots$ & $1.154 \pm 0.033$ \\
\hline$i_{P}(\operatorname{deg}) \ldots \ldots \ldots \ldots \ldots$ & $85.79 \pm 0.24$ \\
\hline 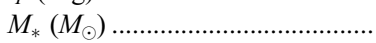 & $0.82 \pm 0.03^{\mathrm{a}}$ \\
\hline 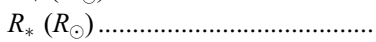 & $0.758 \pm 0.016^{\mathrm{b}}$ \\
\hline 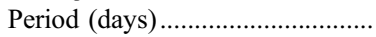 & $2.218573 \pm 0.000020$ \\
\hline 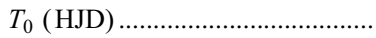 & $2453629.39420 \pm 0.00024$ \\
\hline
\end{tabular}

Parameters from a Simultaneous Fit of Transit Curves

a Mass was fixed; value from B05.
b Radius was fixed; value from Masana et al. (2006).

normalize the relative errors. We also allow the locations of individual transits to vary freely in the fit.

When calculating our transit curves, we use the nonlinear limbdarkening law defined in Claret (2000):

$$
\begin{gathered}
I(r)=1-\sum_{n=1}^{4} c_{n}\left(1-\mu^{n / 2}\right), \\
\mu=\cos \theta .
\end{gathered}
$$

We select the four-parameter nonlinear limb-darkening coefficients from Claret (2000) for a star with $T=5000 \mathrm{~K}, \log g=$ $4.5,[\mathrm{Fe} / \mathrm{H}]=0.0$, and a turbulent velocity of $1.0 \mathrm{~km} \mathrm{~s}^{-1}$. The actual parameters for the star, from B05, are rather close to this: $T=5050 \pm 50 \mathrm{~K}, \log g=4.53 \pm 0.14$, and $[\mathrm{Fe} / \mathrm{H}]=-0.03 \pm 0.04$.

To determine the best-fit radius for the planet, we evaluate the $\chi^{2}$ function over all full transits simultaneously, using the same values for the planetary radius and inclination. For this purpose, we employed the downhill simplex minimization routine AMOEBA from Press et al. (1992). The full transits and the fitted curves are exhibited in Figure 1, and the transit parameters are listed in Table 4. In order to determine the $1 \sigma$ errors, we fit for the inclination and the radius of the planet using the $1 \sigma$ values for the mass and radius of the star (assuming that they are uncorrelated). We find that the mass of the star contributes errors of $\pm 0.004 R_{\mathrm{J}}$ and \pm 0.12 , and the radius of the star contributes errors of $\pm 0.032 R_{\mathrm{J}}$ and \pm 0.21 . Using a bootstrap Monte Carlo method, we also estimate the errors from the scatter in our data and find that this scatter contributes an error of $\pm 0.005 R_{\mathrm{J}}$ and \pm 0.03 to the final measurement. This gives us a total error of $\pm 0.033 R_{\mathrm{J}}$ for the planetary radius and \pm 0.24 for the inclination.

Our best-fit parameters gave a reduced $\chi^{2}$ value of 1.23 . The excess in the reduced $\chi^{2}$ over unity is the result of our method for normalizing the relative errors for data taken at $N_{\mathrm{tr}}=6$, where the rms variation in the data increases significantly toward the end of the data set, as the source moved closer to the horizon. For these data we define our errors as the standard deviation of the data before the transit, where the scatter was much smaller. This is justified because we know from several sources (night Web camera, raw photon counts) that the conditions were similar (photometric) before and during the transit, and the errors before the transit better represent those inside the transit. This underestimates the errors for data after the transit, inflating the $\chi^{2}$ function accordingly. We find that when we exclude the FLWO $1.2 \mathrm{~m}$ data after the end of the transit (the FLWO $1.2 \mathrm{~m}$ data contain significantly more points than any other single data set), the reduced $\chi^{2}$ for the fit decreases to 0.93 .

The results of the planetary transit fit are shown in Table 4 . The value for the radius of the planet, $R_{P}=1.15 \pm 0.03 R_{\mathrm{J}}$, is smaller than the B05 value $\left(R_{P}=1.26 \pm 0.03 R_{\mathrm{J}}\right)$, and the inclination of 
$85^{\circ} .8 \pm 0.2$ is slightly larger than the $\mathrm{B} 05$ value $\left(85^{\circ} .3 \pm 0.1\right)$. Although our errors are comparable to the errors given by B05, despite the superior quality of the new data, we note that this is a direct result of the larger error $( \pm 0.016$ instead of \pm 0.01$)$ for the stellar radius that we use in our fits. As discussed in $\S 3.1$, we feel that this error, which is based on the effective temperature and bolometric magnitude of the star, is a more accurate reflection of the uncertainties in the measurement of the radius of the star. We note that the errors are dominated by the uncertainties in the stellar parameters (notably $R_{*}$ ).

Fitting with unconstrained stellar radius. - We note that when we fit for the stellar radius directly from the transit curves (meaning that we fit for the planetary radius, orbital inclination, and stellar radius, but set the stellar mass equal to $0.82 M_{\odot}$ ), we measure a stellar radius of $0.68 \pm 0.02 R_{\odot}$ and a planetary radius of $R_{P}=1.00 \pm 0.03 R_{\mathrm{J}}$. The errors for these measurements are from a bootstrap Monte Carlo analysis and represent the formal uncertainties in our data alone. (The errors do not change noticeably by incorporating the error from the mass of the star.) These errors do not take into account possible systematics (see below), and they seriously underestimate the real error on the stellar radius, which is very sensitive to the shape of the light curve, and where small distortions in the shape can lead to a large uncertainty.

Nevertheless, it is suggestive that our data prefer a smaller stellar radius (and a correspondingly smaller planetary radius) than our estimates based on temperature, bolometric magnitude, and $V-K$ colors alone would lead us to expect, or a radius smaller than the $0.82 M_{\odot}$ stellar mass implies.

With many more points ( 869 as compared to $\sim 100$ in the other data sets) and lower photon noise uncertainties, the FLWO $1.2 \mathrm{~m}$ data dominate the fit of equation (3) (both the values and the uncertainty of the derived parameters). However, we repeated our fit with and without these data and found that the best-fit radius for the star changed only slightly (to $0.67 R_{\odot}$ ) when the FLWO $1.2 \mathrm{~m}$ data were excluded from the fit. Thus, our $I-, V$-, and $B$-band data independently yield values for the stellar radius that are similar to those implied by the FLWO $1.2 \mathrm{~m}$ data.

The effect of systematic errors. - The $\chi^{2}$ minimization formula (eq. [3]) assumes independent noise, but the presence of covariance in the data (due to systematics in the photometry) means that too much weight may be given to a data set having small formal errors and a great number of data points (e.g., the FLWO $1.2 \mathrm{~m}$ data) compared to the other independent data sets (e.g., other telescopes and filters). This is especially a concern when the data sets yield different transit parameters and one needs to establish whether this difference is significant. In order to follow up with this issue, we repeated the global fit by assuming that the photometric systematics were dominant in the error budget on the parameters, as suggested by our experience with millimagnitude rapid time-series photometry. We estimated the amplitude of the covariance from the variance of 20 minute sliding averages on the residuals around the best-fitting transit light curve for each night, following the method of Pont (2006). The fit was repeated using these new weights (listed in Table 2 as $\sigma_{\text {sys }}$ ), and the resulting parameters (planetary radius, inclination) were within $1 \%$ of the values found assuming independent noise. It is noteworthy that this does not mean the systematics are negligible. The dispersion of these parameters from the individual nights were found to be compatible with the uncertainties due to the systematics.

The amplitude of the systematics is also sufficient to account for the difference in the best-fit stellar radius if it is left as a free parameter. If we fit the OHP, FLWO $1.2 \mathrm{~m}$, HAT-5, HAT-6, and HAT-9 data separately, we get $R_{*}=0.73,0.67,0.55,0.66$, and
$0.57 R_{\odot}$, respectively, with a mean value of $0.64 R_{\odot}$ and a standard deviation of $0.075 R_{\odot}$. This shows that (1) the uncertainty on $R_{*}$ due to the systematics is large and (2) the FLWO $1.2 \mathrm{~m}$ data, by coincidence, are close to the mean of these values; thus, when they were removed from the fit described in the previous paragraph, the results were not changed significantly.

Therefore, with the amplitude of the covariance in the photometry determined from the data themselves, we find that the indications of discrepancy between the different data sets and with the assumed primary radius are not compelling at this point.

\subsection{Ephemerides}

The transit curves derived from the full transits for each bandpass were used in turn to calculate the ephemerides of HD 189733 using all transits that have significant OOT and in-transit sections present (for reference, see Table 2). For each transit (full and partial), the center of transit $T_{C}$ was determined by $\chi^{2}$ minimization. Partial transits with the fitted curve overlaid are exhibited in Figure 2. Errors were assigned to the $T_{C}$ values by perturbing $T_{C}$ so that $\chi^{2}$ increases by unity. The individual $T_{C}$ transit locations and their respective errors are listed in Table 5. The typical timing errors were formally on the order of 1 minute. This, however, does not take into account systematics in the shape of the light curves. The errors in $T_{C}$ can be estimated from the simultaneous transit observations; for example, the $N_{\text {tr }}=6$ event was observed by the FLWO $1.2 \mathrm{~m}$, HAT-5, HAT-6, and TopHAT telescopes (Table 2), and the rms of $T_{C}$ around the median is $\sim 50 \mathrm{~s}$, which is in harmony from the above independent estimate of 1 minute. We applied an error-weighted least-squares minimization on the $T_{C}=P N_{\text {tr }}+E$ equation, where the free parameters were the pe$\operatorname{riod} P$ and the epoch $E$. The refined ephemeris values are listed in Table 4. They are consistent with those derived both by B05 and by Hébrard \& Lecavelier Des Etangs (2006), using Hipparcos and OHP $1.2 \mathrm{~m}$ data, to within $1 \sigma$ using our error bars.

We also examined the observed minus calculated $(O-C)$ residuals, as their deviation can potentially reveal the presence of moons or additional planetary companions (Holman \& Murray 2005; Agol et al. 2005). The $O-C$ values are listed in Table 4 and plotted in Figure 3. Using the approximate formula from Holman \& Murray (2005), ${ }^{13}$

$$
\begin{aligned}
\Delta t & \sim \frac{45}{16 \pi}\left(\frac{M_{2}}{M_{*}}\right) P_{1} \alpha_{e}^{3}\left(1-\sqrt{2} \alpha_{e}^{3 / 2}\right)^{-2}, \\
\alpha_{e} & =\frac{a_{1}}{a_{2}\left(1-e_{2}\right)}
\end{aligned}
$$

as an example, a $0.15 M_{\mathrm{J}}$ perturbing planet on a circular orbit at 2 times the distance of HD 189733 b $(P \approx 6.3$ days, not in resonance) would cause variations in the transit timings of $15 \mathrm{~s}$. The radial velocity semiamplitude of HD 189733 as induced by this hypothetical planet would be $19 \mathrm{~m} \mathrm{~s}^{-1}$, which would be barely noticeable (at the $1 \sigma$ level) from the discovery data, having residuals of $15 \mathrm{~m} \mathrm{~s}^{-1}$ and spanning only 30 days.

A few seemingly significant outlier points on the $O-C$ diagram are visible, but we believe that it would be premature to draw any conclusions, because (1) the error bars do not reflect the effect of systematics, and, for example, the $T_{C}$ of the $N_{\mathrm{tr}}=0$ OHP discovery data moved by $\sim 5$ minutes after recalibration of that data set, and (2) all negative $O-C$ outliers are $B$ - or $V$-band data, which is suggestive of an effect of remaining color-dependent

\footnotetext{
13 The original equation had an error, and $\pi$ has been moved to the denominator.
} 
TABLE 5

Best-Fit Transit Locations

\begin{tabular}{|c|c|c|c|c|c|}
\hline Telescope & $N_{T}$ & $\begin{array}{c}T_{C} \\
\text { (HJD) }\end{array}$ & $\begin{array}{c}\sigma_{\mathrm{HJD}} \\
\text { (days) }\end{array}$ & $\begin{array}{l}O-C \\
\text { (days) }\end{array}$ & $(O-C) / \sigma_{\mathrm{HJD}}$ \\
\hline OHP $1.2 \mathrm{~m} . \ldots \ldots \ldots \ldots \ldots . . . . . . . . .$. & 0 & 2453629.39073 & \pm 0.00059 & -0.0035 & -5.9 \\
\hline 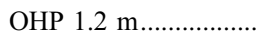 & 4 & 2453638.26885 & \pm 0.00067 & 0.00035 & 0.53 \\
\hline OHP $1.2 \mathrm{~m}$ & 5 & 2453640.48706 & \pm 0.00174 & -0.0000079 & -0.0045 \\
\hline FLWO $1.2 \mathrm{~m} \ldots \ldots \ldots . . . . .$. & 6 & 2453642.70592 & \pm 0.00022 & 0.00029 & 1.24 \\
\hline HAT-5 ........................... & 6 & 2453642.70641 & \pm 0.00092 & 0.00077 & 0.84 \\
\hline HAT-6 & 6 & 2453642.70649 & \pm 0.00049 & 0.00085 & 1.7 \\
\hline 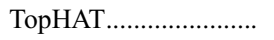 & 6 & 2453642.70536 & \pm 0.00048 & -0.00028 & -0.57 \\
\hline HAT-9 ..................... & 7 & 2453644.92720 & \pm 0.00111 & 0.0030 & 2.7 \\
\hline HAT-9 ................... & 16 & 2453664.89287 & \pm 0.00108 & 0.0015 & 1.4 \\
\hline HAT-5 ........................ & 19 & 2453671.54999 & \pm 0.00113 & 0.0029 & 2.6 \\
\hline 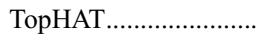 & 19 & 2453671.54849 & \pm 0.00096 & 0.0014 & 1.5 \\
\hline HAT-5 ............................ & 20 & 2453673.76725 & \pm 0.00072 & 0.0016 & 2.2 \\
\hline 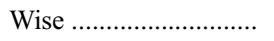 & 22 & 2453678.20080 & \pm 0.00050 & -0.0020 & -4.0 \\
\hline 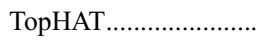 & 24 & 2453682.63715 & \pm 0.00100 & -0.0028 & -2.8 \\
\hline HAT-9 ........................ & 29 & 2453693.73327 & \pm 0.00090 & 0.00045 & 0.51 \\
\hline
\end{tabular}

Notes.-These are the best-fit locations for the centers of the fifteen full and partial eclipses examined in this work. We also give the number of elapsed transits $N_{T}$ and $O-C$ residuals for each eclipse.

systematics. The significance of a few outliers is further diminished by the short data set we have; no periodicity can be claimed by observing two full and nine partial transits altogether.

According to the theory, the nature of perturbations would be such that they appear as occasional, large outliers. Thus, the detection of potential perturbations also benefits from the study of numerous sequential transits; for example, the Microvariablity and Oscillations of Stars (MOST) mission with continuous coverage and uniform data would be suitable for such study (Walker et al. 2003). We also draw the attention to the importance of observing full transits, as they improve the $T_{C}$ center of transit by a significant factor, partly because of the presence of ingress and egress, and also due to a better treatment of the systematics.

If a planet is perturbed by another, outer planet (and they are not in resonance), the transit-time variations $\Delta t$ are proportional to the period $P$ of the perturbed planet (Agol et al. 2005). Although HD $189733 b$ is a relatively short-period (2.21 days) planet

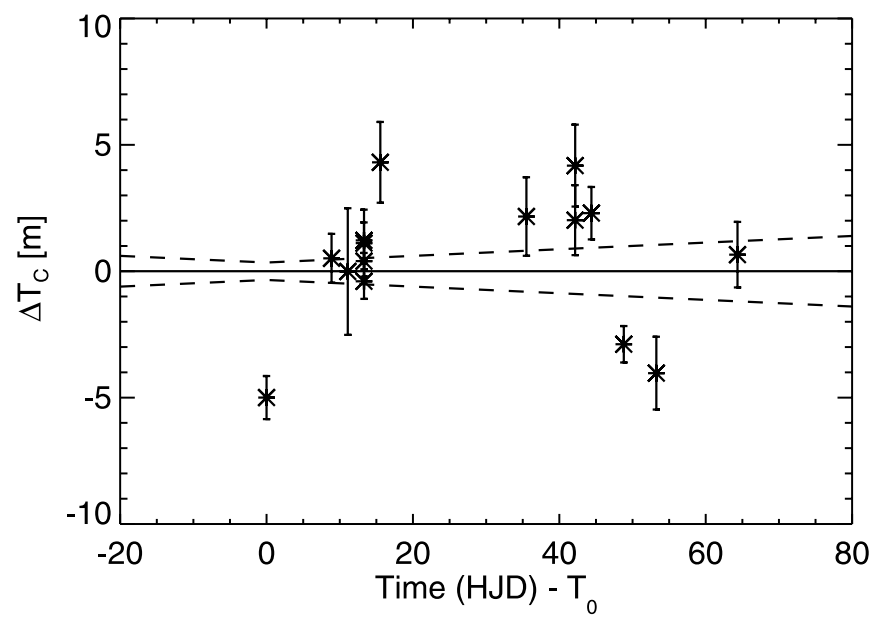

FIG. 3.- Residuals calculated using the values of the period and $T_{0}$ derived in this work. The dashed lines are calculated from the uncertainties in the measurements of $P$ and $T_{0}$. The figure in the electronic edition is color-coded according to the bandpass used. [See the electronic edition of the Journal for a color version of this figure.] compared to, for example, HD 209458b (3.5 days), it is a promising target for detecting transit perturbations in the future, because the mass of the host star is low and $\Delta t \propto 1 / M_{*}$; plus, the deep transit of the bright source will result in very precise timing measurements. Observations spanning several months to many years may be needed to say anything definite about the presence or absence of a periodic perturbation.

\section{CONCLUSIONS}

Our final values for the planetary radius and orbital inclination were derived by fixing the stellar radius and mass to independently determined values from B05 and Masana et al. (2006). We analyzed the data set in two ways: by $\chi^{2}$ minimization assuming independent errors, and also by assuming that photometric systematics were dominant in the error budget. Both methods yielded the same transit parameters within 1\%: if we assume that there is no additional unresolved close-in stellar companion to HD 189733 to make the transits shallower, then we find a planetary radius of $1.154 \pm 0.033 R_{\mathrm{J}}$ and an orbital inclination of $85^{\circ} .79 \pm 0.24$ (Table 4). The uncertainty in $R_{P}$ is primarily due to the uncertainty in the value of the stellar radius.

We note that the TopHAT $V$-band full- and partial-transit data, as well as the Wise partial-transit $B$-band data, appear slightly deeper than the best fit to the analytic model. The precision of the data set is not adequate to determine if this potential discrepancy is caused by a real physical effect (such as a second stellar companion) or to draw further conclusions.

When compared to the discovery data, the radius decreased by $10 \%$, and HD $189733 \mathrm{~b}$ is in the mass and radius range of "normal" exoplanets (Fig. 4). The decrease is due to the new, superior quality data set that originates from many telescopes and covers multiple photometric bandpasses. It also includes a more careful rereduction of the discovery data. The revised radius estimate is consistent with structural models of hot Jupiters that include the effects of stellar insolation, and hence it does not require the presence of an additional energy source, as is the case for HD $209458 \mathrm{~b}$. On the mass-radius diagram, HD 209458b remains an outlier with anomalously low density. We note that the parameters of OGLE-10b are still debated (Konacki et al. 2005; Holman et al. 2005; Santos et al. 2006), but according to the recent analysis 


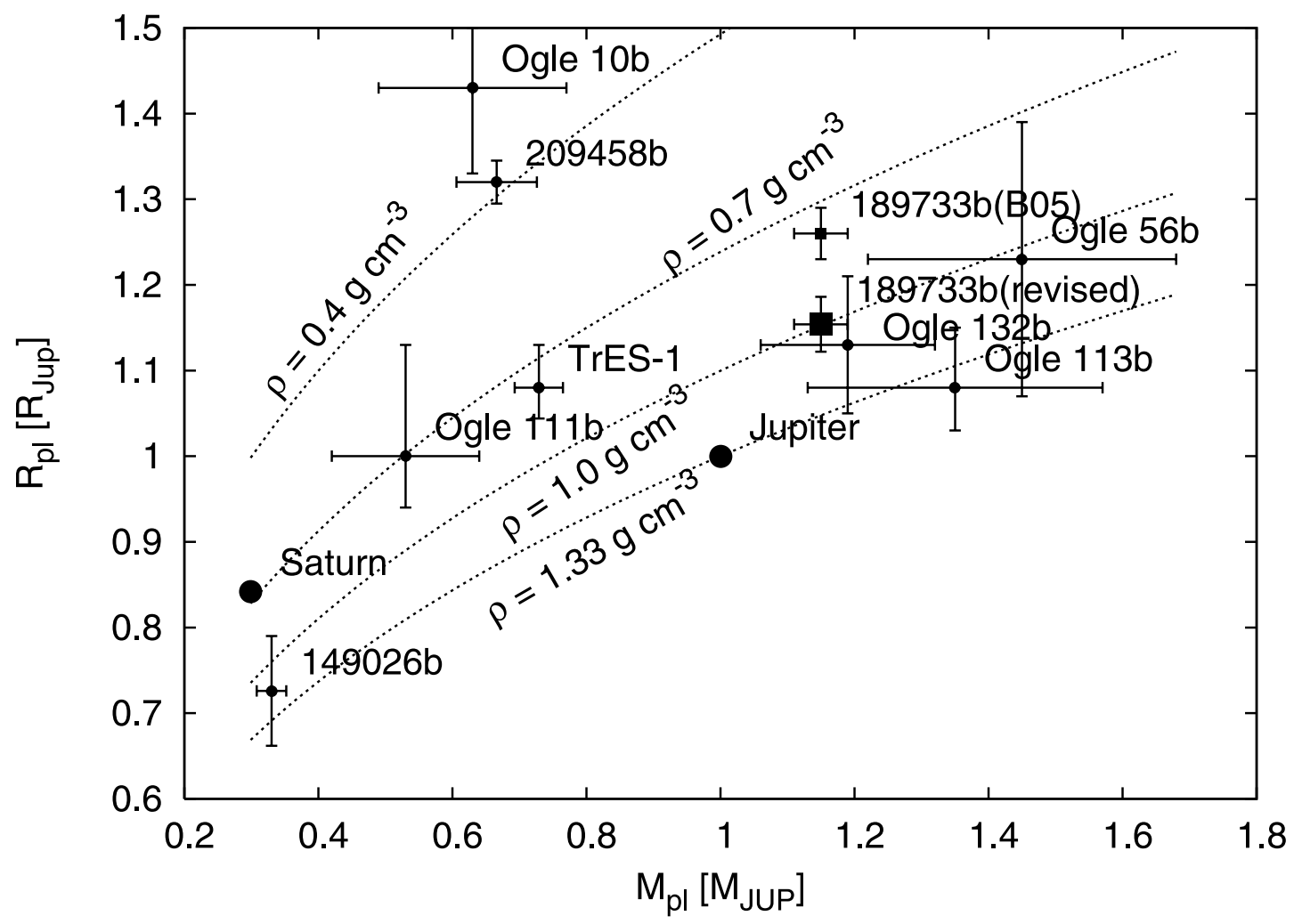

FIG. 4.- Mass-radius diagram depicting known transiting exoplanets, plus Saturn and Jupiter (for comparison). Both the discovery (B05; small filled square) and revised radius (large filled square) for HD 189733 are shown. Sources for the mass and radius values (in that order) are listed after the name of the planet: HD 209458b: Laughlin et al. (2005), Knutson et al. (2006); HD 189733: B05, this paper; OGLE 111b: Pont et al. (2004); OGLE 10b: Konacki et al. (2005), Santos et al. (2006); OGLE 132b: Moutou et al. (2004); OGLE 56b: Torres et al. (2004); OGLE 113b: Bouchy et al. (2004); TrES-1: Laughlin et al. (2005); HD 149026: Sato et al. (2005), Charbonneau et al. (2006).

of Santos et al. (2006), it also has anomalously low density. With its revised parameters, HD $189733 \mathrm{~b}$ is quite similar to OGLETR-132b (Moutou et al. 2004). The smaller radius leads to a higher density of $\sim 1 \mathrm{~g} \mathrm{~cm}^{-3}$, as compared to the former measurement of $\sim 0.75 \mathrm{~g} \mathrm{~cm}^{-3}$. The smaller planetary radius increases the $16 \mu \mathrm{m}$ brightness temperature $T(16 \mu \mathrm{m})=1117 \pm$ $42 \mathrm{~K}$ of Deming et al. (2006) to $1279 \pm 90 \mathrm{~K}$, which is slightly larger than that of TrES-1 and HD 209458b.

We also derived new ephemerides and investigated the outlier points in the $O-C$ diagram. We have not found any compelling evidence for outliers that could be due to perturbations from a second planet in the system. We note, however, that due to the proximity and brightness of the parent star, as well as the deep transit, the system is well suited for follow-up observations.
Part of this work was funded by NASA grant NNG04GN74G. Work by G. Á. B. was supported by NASA through grant HSTHF-01170.01-A (Hubble Fellowship). H. K. is supported by a National Science Foundation Graduate Research Fellowship. D. W. L. thanks the Kepler mission for support through NASA Cooperative Agreement NCC2-1390. A. P. wishes to acknowledge the hospitality of the Harvard-Smithsonian Center for Astrophysics, where part of this work has been carried out. Work of A. P. was also supported by Hungarian OTKA grant T-038437. Research of T. M. and A. S. was partially supported by the GermanIsraeli Foundation for Scientific Research and Development. This publication makes use of data products from the Two Micron All Sky Survey (2MASS). We thank M. Hicken and R. Kirshner for swapping nights on the FLWO $1.2 \mathrm{~m}$ telescope on short notice.

\section{REFERENCES}

Agol, E., Steffen, J., Sari, R., \& Clarkson, W. 2005, MNRAS, 359, 567

Bakos, G. Á., Lázár, J., Papp, I., Sári, P., \& Green, E. M. 2002, PASP, 114, 974

Bakos, G. Á., Noyes, R. W., Kovács, G., Stanek, K. Z., Sasselov, D. D., \& Domsa, I. 2004, PASP, 116, 266

Bakos, G. Á., Pál, A., Latham, D. W., Noyes, R. W., \& Stefanik, R. P. 2006, ApJ, 641, L57

Baraffe, I., Chabrier, G., Allard, F., \& Hauschildt, P. H. 1998, A\&A, 337, 403

Baraffe, I., Chabrier, G., Barman, T. S., Selsis, F., Allard, F., \& Hauschildt, P. H. 2005, A\&A, 436, L47

Bessell, M. S., Castelli, F., \& Plez, B. 1998, A\&A, 333, 231

Bouchy, F., Pont, F., Santos, N. C., Melo, C., Mayor, M., Queloz, D., \& Udry, S. 2004, A\&A, 421, L13

Bouchy, F., et al. 2005, A\&A, 444, L15 (B05)

Carpenter, J. M. 2001, AJ, 121, 2851

Charbonneau, D., et al. 2006, ApJ, 636, 445

Claret, A. 2000, A\&A, 363, 1081
Cox, A. N., ed. 2000, Allen's Astrophysical Quantities (4th ed.; New York: AIP)

Deming, D., Harrington, J., Seager, S., \& Richardson, L. J. 2006, ApJ, 644, 560 Gilliland, R. L., \& Brown, T. M. 1988, PASP, 100, 754

Girardi, L., Bertelli, G., Bressan, A., Chiosi, C., Groenewegen, M. A. T., Marigo, P., Salasnich, B., \& Weiss, A. 2002, A\&A, 391, 195

Hébrard, G., \& Lecavelier Des Etangs, A. 2006, A\&A, 445, 341

Holman, M. J., \& Murray, N. W. 2005, Science, 307, 1288

Holman, M. J., Winn, J. N., Stanek, K. Z., Torres, G., Sasselov, D. D., Allen,

R. L., \& Fraser, W. 2005, ApJL, submitted (astro-ph/0506569)

Kervella, P., Thévenin, F., Di Folco, E., \& Ségransan, D. 2004, A\&A, 426, 297 Knutson, H., Charbonneau, D., Noyes, R. W., Brown, T. M., \& Gilliland, R. L. 2006, ApJ, submitted (astro-ph/0603542)

Konacki, M., Torres, G., Sasselov, D. D., \& Jha, S. 2005, ApJ, 624, 372

Konacki, M., et al. 2004, ApJ, 609, L37

Landolt, A. U. 1992, AJ, 104, 340 
Laughlin, G., Wolf, A., Vanmunster, T., Bodenheimer, P., Fischer, D., Marcy, G., Butler, P., \& Vogt, S. 2005, ApJ, 621, 1072

Mandel, K., \& Agol, L. 2002, ApJ, 580, L171

Masana, E., Jordi, C., \& Ribas, I. 2006, A\&A, 450, 735

Moutou, C., Pont, F., Bouchy, F., \& Mayor, M. 2004, A\&A, 424, L31

Newberry, M. V. 1991, PASP, 103, 122

Perryman, M. A. C., et al. 1997, A\&A, 323, L49

Pont, F. 2006, in Tenth Anniversary of 51 Peg-b, ed. L. Arnold, F. Bouchy, \& C. Moutou (Paris: Frontier Group), 153

Pont, F., Bouchy, F., Melo, C., Santos, N. C., Mayor, M., Queloz, D., \& Udry, S. 2005, A\&A, 438, 1123

Pont, F., Bouchy, F., Queloz, D., Santos, N. C., Melo, C., Mayor, M., \& Udry, S. 2004, A\&A, 426, L15

Press, W. H., Teukolsky, S. A., Vetterling, W. T., \& Flannery, B. P. 1992, Numerical Recipes (2nd ed.; Cambridge: Cambridge Univ. Press)

Santos, N. C., et al. 2006, A\&A, 450, 825
Sato, B., et al. 2005, ApJ, 633, 465

Skrutskie, M. F., et al. 2006, AJ, 131, 1163

Stetson, P. B. 1987, PASP, 99, 191

Tody, D. 1986, Proc. SPIE, 627, 733

1993, in ASP Conf. Ser. 52, Astronomical Data Analysis Software and Systems II, ed. R. J. Hanisch, R. J. V. Brissenden, \& J. Barnes (San Francisco: ASP), 173

Torres, G., Konacki, M., Sasselov, D. D., \& Jha, S. 2004, ApJ, 609, 1071

Udalski, A., Szewczyk, O., Żebruń, K., Pietrzyński, G., Szymański, M., Kubiak, M., Soszyński, I., \& Wyrzykowski, Ł. 2002a, Acta Astron., 52, 317

Udalski, A., Żebruń, K., Szymański, M., Kubiak, M., Soszyński, I., Szewczyk, O., Wyrzykowski, Ł., \& Pietrzyński, G. 2002b, Acta Astron., 52, 115

Udalski, A., et al. 2002c, Acta Astron., 52, 1

Walker, G., et al. 2003, PASP, 115, 1023

Wittenmyer, R. A., et al. 2005, ApJ, 632, 1157

Young, A. T. 1967, AJ, 72, 747 